\title{
Towards Open Agent Systems Through Dynamic Incorporation
}

\author{
C. Cubillos, M. Donoso, N. Rodríguez, F. Guidi-Polanco, D. Cabrera-Paniagua
}

\author{
Claudio Cubillos, Makarena Donoso \\ Nibaldo Rodríguez, Franco Guidi-Polanco \\ Pontificia Universidad Católica de Valparaíso \\ Av. Brasil 2241, Valparaíso, Chile \\ E-mail: \{claudio.cubillos,nibaldo.rodriguez,fguidi\}@ucv.cl \\ makarena.donoso@gmail.com
}

Daniel Cabrera-Paniagua

Universidad de Valparaíso

Valparaíso, Chile

E-mail:daniel.cabrera@uv.cl

\begin{abstract}
This work tackles the problem of providing a mechanism and infrastructure for allowing a given Multiagent System (MAS) to become open, allowing the incorporation of newly incoming agents to participate within the existing society. For this, a conceptual analysis of the so-called conciliation problem is presented, covering the diverse levels and issues involved in such a process. Our Dynamic Incorporation Architecture is presented, which implements an infrastructure for allowing the participation of external agents into a specific multiagent system by incorporating the appropriate behaviours upon arrival. Our multiagent architecture for dynamic incorporation covers three levels: semantics, communication and interaction and has been applyed in a book-trading e-market scenario.
\end{abstract}

Keywords: Multiagent System, Dynamic Incorporation Architecture, Open Agent Systems, PASSI.

\section{Introduction}

Software agents are defined as autonomous entities capable of flexible behavior denoted by reactiveness, pro-activeness and social ability [1]. Multiagent systems (MAS) consist of diverse agents that communicate and coordinate generating synergy to pursue a common goal. At present, Multiagent Systems (MAS) raises as a key paradigm for the development of next generation software systems which are required to be distributed, intelligent (autonomous, proactive), open and dynamic. While much work has been done by the research community in solving distribution and intelligence issues, little effort has been devoted to openness and dynamicity under MAS settings.

In the medium-to-long-term future we will see open multi-agent systems spanning multiple application domains, and involving heterogeneous participants developed by diverse design teams. Agents seeking to participate in these systems will be able to incorporate and learn the appropriate behavior for participation in the course of doing so, rather than having to prove adherence before entry (as happens today) [2].

However, up to now agent systems typically center on closed agent systems with ad-hoc designs and predefined communications protocols. In recent years, agent systems have evolved to the use of agreed protocols and languages thanks to a huge standardization effort (FIPA, OMG, W3C). Nowadays agent system openness is limited to the participation of any agent able to satisfy publicly-advertised standards. Moreover, typically communication protocols, languages 
(ACLs) and domain knowledge model (ontology) are defined by the design team prior to any agent interactions. Therefore much work needs to be done for the above scenario to become true.

The current work presents a conceptual analysis of the so-called conciliation problem for then presenting the design of a multiagent architecture devoted to facilitate the dynamic participation of external agents into a specific multiagent system by incorporating the appropriate behavior upon arrival. A book-trade e-market has been used as study case to validate the multiagent implementation. This work gives continuity to our previous research in [4] [5].

\section{Related Work}

Relevant research has been developed around coalition formation; the process to form a group of agents and solve a problem via cooperation [4], some works on Dynamic Coalition Formation (DCF) [5] tackle the issue of dynamically building beneficial coalitions (coalition algorithms) among agents that can cope with environmental changes without restarting the negotiation process.

However, at present coalition formation for virtual organizations is limited, with such organizations being largely static. All of the existing work has been devoted to optimizing for a given agent, the decision of when to participate or not in a coalition (conformation/disband) but not in providing an infrastructure supporting such dynamic coalition conformations and their agents' heterogeneities.

The novelty of our work relies on 1) presenting a conceptual approach for conciliating agents' heterogeneities under an open MAS setting 2) present a solution based in dynamic behavior loading rather than in a mediated-architecture approach and 3) implementing a solution that solves heterogeneities at two levels: semantics and interaction (behavior) while leaving the implementation of the communication level (related services and conciliation)as further work.

\section{The Three Conciliation Levels}

For the Dynamic formation of agent systems to become true, a set of diverse issues need to be solved first. This concern: 1) The mechanism needed to provide the incorporation of a foreign agent to the society (agent system), 2) The ways to allow the agent to incorporate upon-arrival the society common knowledge (ontology) in order to interact, and 3) The means allowing the foreign agent to learn or incorporate upon-arrival the communication protocols and languages used by the society (AIPs [6], ACLs [7], etc) and the inherent business model.

Each of the above issues regards a different aspect of the communication infrastructure used by the agent society to interact and each one is covered by different areas of informatics and computer science. In general terms, the problem of conciliating the existing divergences on the communication capabilities of the entering agent and the existing society can be organized into three levels: firstly, the interaction level, involving the diverse agent interaction protocols (AIP's) to formalize the conversations among agents. Then the communication level, tackling the heterogeneinity at message level, that is, the protocol and language used on the messages. Finally, the semantic level, conciliating the possible divergences on the symbols used in the messages and the underlying knowledge models of the new agent and the MAS society. Each of these is further explained in the following.

\subsection{Interaction Level}

The highest level is the interaction one, tackling the conversations among pair of agents through agent interaction protocols (AIP's) that specify the underlying coordination / coopera- 
tion mechanism. Examples can be the contract-net protocol (CNP) [11], the different auctions (e.g. English, Dutch, Vickrey, etc.), tuple-based negotiations, among others. These specific protocols are grounded into interaction diagrams that specify the roles of the participants and the expected messages to be sent and received under which conditions. In practical terms, the new agent will need to incorporate the behavior needed to perform a certain role within the MAS society. For example, turning back to the cooper e-market, the new agent will need to add or load the manager role for initiating a contract-net, which will allow him to make the call for proposals, evaluate and select the best proposal and award it. All these tasks will need to be incorporated at run-time by simply instantiating the corresponding behavior classes and adding it to the agent.

By having this solve, the agent will still need to understand the parameters required by each of the tasks it has loaded, moving us to the semantic level. Other alternative is to have the agent with the correct interaction protocol and role (e.g. manager of a contract-net) but have divergences on the message format or language used in the content, moving us to the Communication level. Both are described below.

\subsection{Communication Level}

Nowadays agent systems do use communication protocols of their choice that define the message structure or syntax and language used to express the content of the message. Examples of such can be FIPA ACL [8], KQML, an ad-hoc XML-based message envelope, or even the simple but effective concatenization of the different data in an specific order (e.g such as in lowlevel protocols). It can happen that the new agent does know the FIPA ACL Message format but not the Prolog language used in the content of the message or can do not know both. Now when considering the newly incoming agent, how can we enable the incorporation of those message formats and content language upon entry?

One scenario is when the new agent has already the adequate roles and behavior to interact (interaction level) but with a message format and content language that is different from the ones used within the agent society. In this case, a mediation service can be provided, by establishing the mapping of the different slots used on the two message formats. A more efficient approach is the use of a meta-format (meta-ontology for communication) used as message interchange format [9]. In this way each new format must provide its mapping to the meta-format and vice-versa instead of providing its mapping to all the other existing formats.

Other scenario is when the new agent does not have incorporated the interaction role nor the corresponding behaviors. In this case are directly adopted the format for messages and language used by the society when incorporating the behaviors, that is, when solving the problems at interaction level.

\subsection{Semantic Level}

Existing coordination strategies rely on standard interaction and communication protocols, assuming that all participants (agents) understand the shared domain knowledge usually modelled in terms of an ontology. This assumption is no longer valid in open systems in which an incoming agent (usually developed by another party and in another moment) needs to interact with a certain agent society to obtain some service (pursuing a specific goal) and does not know in advance such domain knowledge used to interact. Some questions to answer are: How can the agent request or get that ontology from the agent society and process it or understand it? or how can the agent society provide the incoming agent with all the required knowledge.

The basic problem here is how to conceal the concepts of the new agent with regard to the ones used by the society. Therefore the entering agent will need to be provided by a service 
capable of aligning his knowledge models with the one of the MAS society, requiring ontology alignment and mapping techniques to solve it.

\section{Dynamic Participation Architecture}

This project used PASSI (Process for Agent Societies Specification and Implementation) as methodology of development, which uses UML as modelling language. For a detailed description please refer to [3]. Figure 1 Shows our conceived solution in terms of the conciliation levels described before.

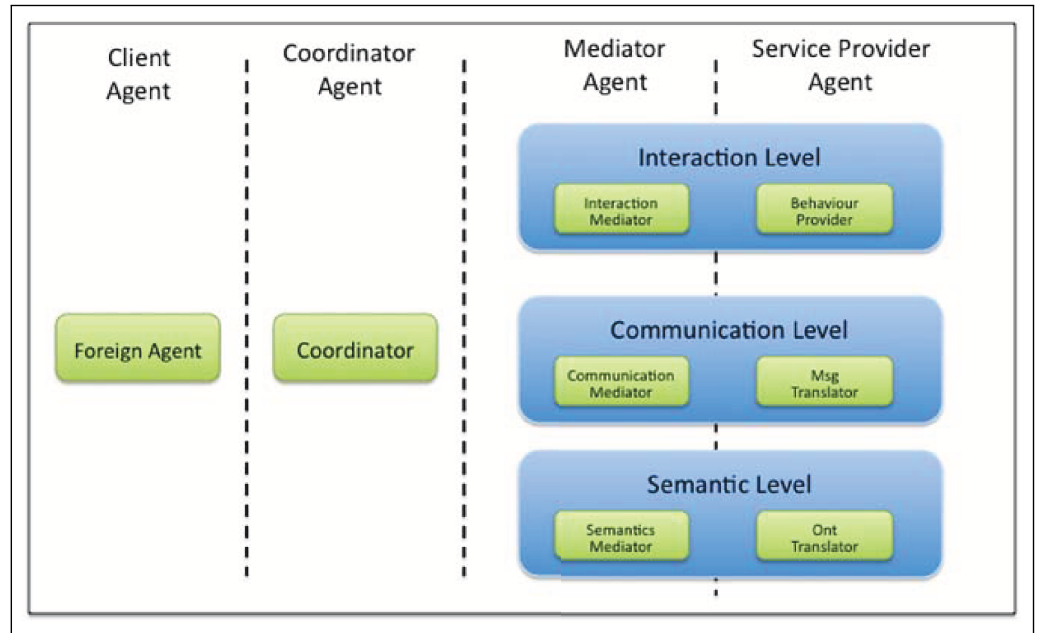

Figure 1: The 3-Tier architecture for conciliation

Firstly, the Foreign Agent (FA) is the one that wants to participate in a specific MAS to which it does not belong beforehand, thus requiring a conciliation process. A Coordinator manages the diverse steps in the agent incorporation from the initial request until the agent is ready to start interacting within the MAS society. Another type of agent is the Mediator, existing one for tackling each of the three levels of possible divergences (semantic, communication and interaction). In the first two cases, each one will have subscribed a set of Translator agents providing specific bridging services among a couple of ontologies, or a pair of message formats in correspondence with the level. In the case of the Interaction Mediator, it will be having associated a set of Behavior-Provider agents, each one containing the code for the roles in diverse interaction protocols (e.g. manager/bidder in Contract Net, auctioneer/auctionee in Auctions, etc.).

The Figure 2 shows when a new agent requests incorporation to the Book-trading MAS. For this, the FA agent sends a conciliation request to the Coordinator containing: the MAS domain name to which the FA wants to contact (Booktrading in our case); the identifier of the message protocol that the FA has; the ID of ontology that the FA knows; and the role name that the FA wants to perform within the Book-trading MAS. Such request is evaluated by the Coordinator checking whether if the FA needs conciliation services at any of the three considered levels; be provided with appropriate behaviors to participate (interaction), message-protocol translators (communication) and ontology translators (semantic). for this it compares the protocols and semantics used by the agent and by the domain MAS searching for heterogeneities.

The coordinator derivates to the Interaction Mediator the search for the requested role and related coded-behaviors. On its turn, the mediator contacts specific Behavior providers following the Contract-Net Protocol [11]. A similar process is carried out for the Communication Mediator and Semantic Mediator. In the first case, the Coordinator sends the ID of the message protocol 


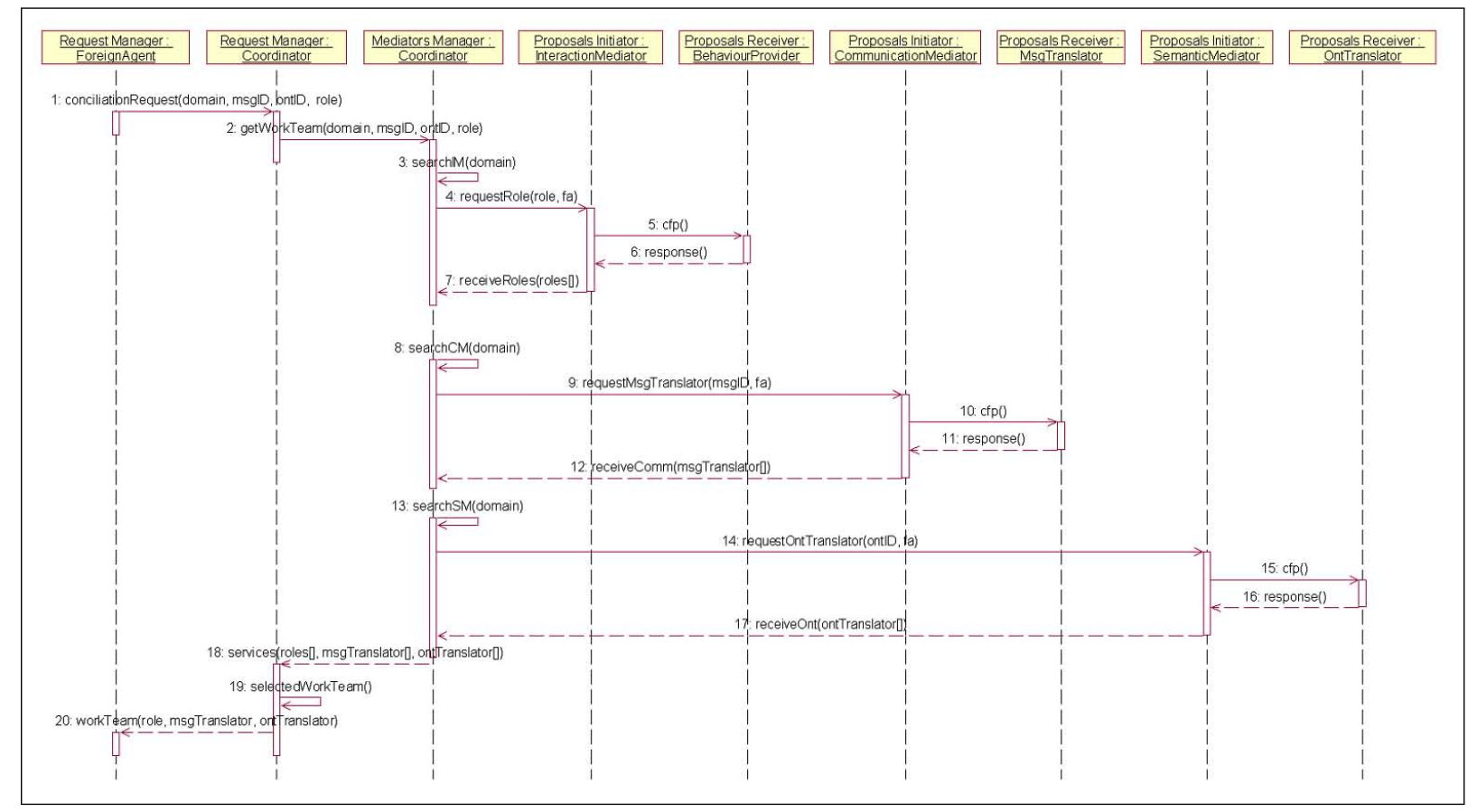

Figure 2: Scenario showing when a Foreign Agent requests a conciliation

used by the FA and the domain MAS, while in the second, sends the ontologies ID of the domain MAS and FA. In all three cases what is received is a list of possible providers and their proposals(bids), as the Coordinator is the one incharge of selecting the final providers, composing the Work Team and announcing it to the FA.

For selecting the appropriate members of the Work Team, the Coordinator should consider diverse aspects such as the cost, quality of service, reliability, etc. of each candidate according to its utility function. On its actual form, the Coordinator selects the providers based on the cost of each service. More variables within the objective function together with other selection schemas (e.g. foreign agent selection or a mixed approach) remains a matter of future work.

The conciliation process may result in the need of services with any combination of the three levels or even require no services in case of a perfect match. In this way, the Coordinator has the responsibility of structuring an appropriate Work Team for the FA. Usually, the Coordinator will select an OntologyTranslator (OT), a MessageTranslator (MT), and a BehaviorProvider (BP) agent.

It is important to mention that each role that the FA agent wants to perform needs a specific Work Team, therefore are tackled as different conciliation requests. However, nothing prevents a service provider of participating in diverse work teams, even for a same foreign agent. Together with requesting and selecting an appropriate work team for the Foreign Agent, another important process is the actual enforcement of those services. In practical terms, this means sending the behaviors from the provider to the FA, plus the possible translation services at communication and semantic level that could be needed.

The Figure 3 shows the services' enforcement, beginning with the Foreign Agent which decomposes the received working team. It gets the behaviors from the selected Behavior Provider while obtaining the corresponding codecs from the Message and Ontology Translators. Upon receiving the behaviors that compose the role, the FA takes the list of parameters required by the behaviors to work and translates it with the Ontology codec. This codec translates the concepts from the domain-MAs ontology to the FA ontology. In this way, the FA can map the values of 


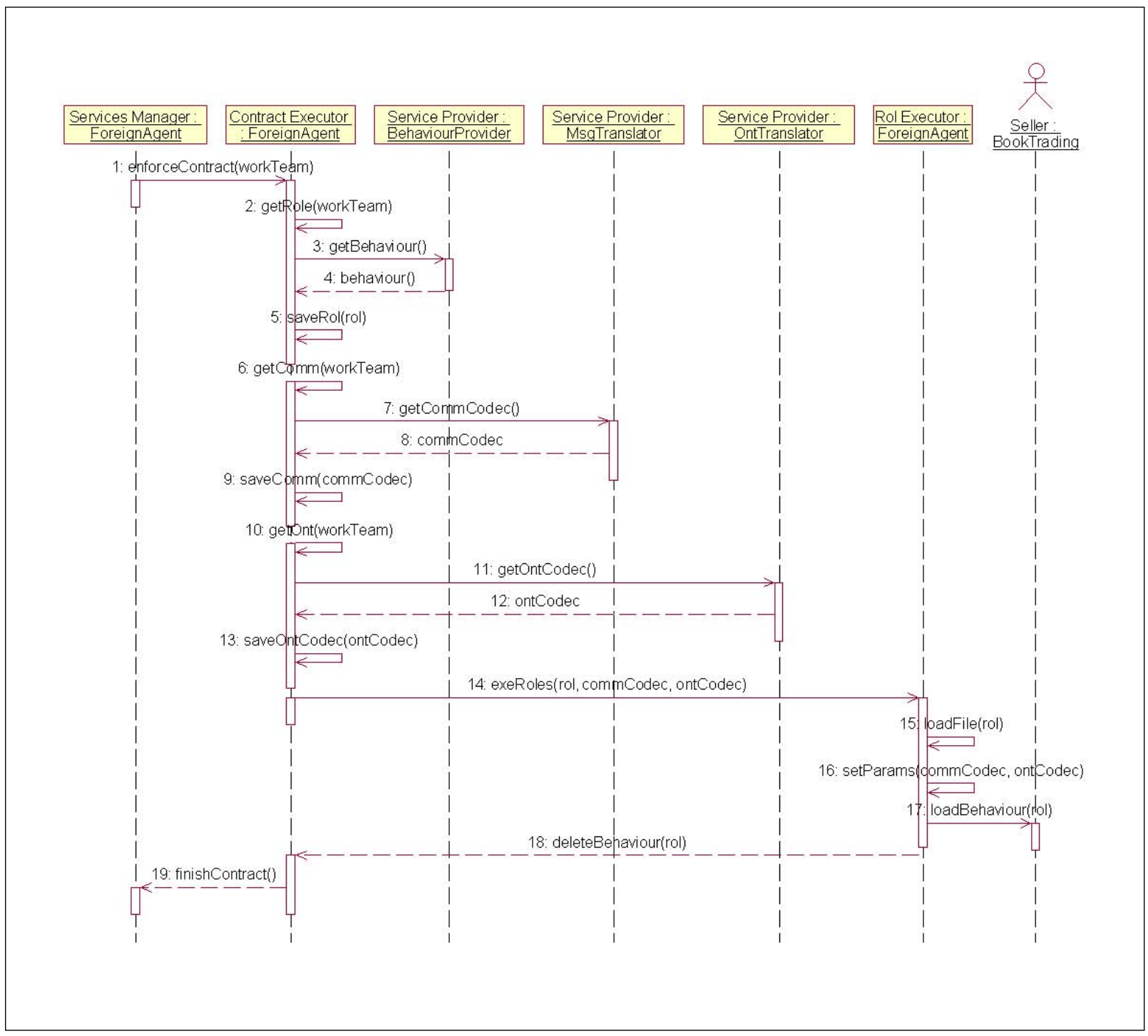

Figure 3: Scenario showing a Foreign Agent in the Contract enforcing process

the behavior parameters with its own attributes and provide the necessary arguments to them. On its turn, the communication codec is used to translate all the inbound/outbound messages among the message protocols used inside the behavior and by the domain MAS.

Regarding the Work Team composition, in a most general case we will be having several ways of composing types of BPs, CMs and STs to conciliate a same FA-to-target-MAS situation. However, due to the combinatorial character of the problem a simplification has been made considering only one possible combination of providers' types. Furthermore, the FA will usually need conciliation at two levels: the interaction and semantics, as usually the behaviors provided will already use the communication protocols employed by the target MAS.

\section{Book-Trading Scenario}

The study case is based on the well-known book trading MAS environment in which Seller and Buyer agents pursue their goals through the Contract-Net Protocol (CNP) [11]. The book-trading example included in the JADE development has been used as baseline. Buyer agents initiate 


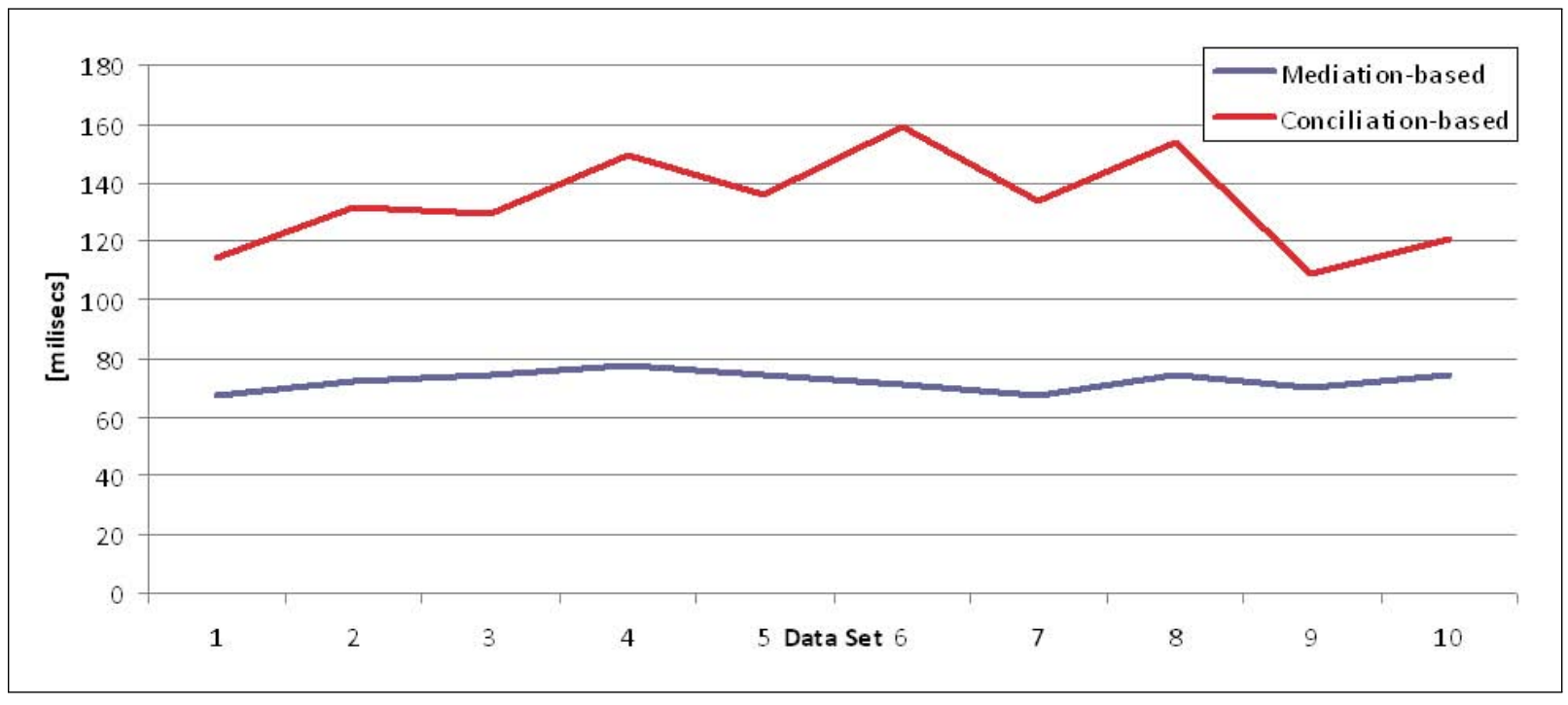

Figure 4: Performance comparison of the mediation-based and conciliation-based architectures

the interaction with a call-for-bids, adopting the Manager role of the CNP. The call includes the book title the buyer is looking for. On the other side, Seller agents assume as potential contractors, processing the calls and making bids for the requested title. In our implementation, the seller looks in its database for the title and sends a bid in case of having stock. The price within the bid is specific for each Seller. The buyer collects the answers and selects the cheapest one.

\section{$5.1 \quad$ Experiments and Results}

The objective of the study case was to prove the feasibility of our Dynamic Participation Architecture and evaluate the associated costs mainly in terms of performance. For this, the experiments focused in comparing a traditional book-trading market (as the one above) with a mediation-based approach and with our proposal. In the first case, Buyers and Sellers interact through a Mediator agent while in the last case, buyer agents correspond to Foreign Agents willing to dynamically participate in the book-trading MAS by adopting upon arrival the required behavior. In this particular case, the role requested for conciliation is manager of the CNP with its diverse tasks (e.g. call for proposal, bid selection, task awarding, etc.)

The test scenario has been generated through a random generation of book requests from a list of 30 titles. The number of sellers has been fixed to 10, each of which has one unit of each book in the list at a price that distributes uniformly U[50, 120]. A total of 10 sets of 100 requests has been generated. The simulation considers a main agent devoted to managing the creation of buyer agents, seller agents and the MAS for dynamic participation. The generation and arrival of buyer agents follows a Poisson distribution, hence the time between arrivals distributes Exponential, $E(\lambda)$, with $\lambda=2$ in terms of requests per second.

For more details on the architecture design and PASSI-UML diagrams please refer to [5]. Regarding the considered distributed environment, the simulations were carried out over PCs with Intel Pentium 4 of $2 \mathrm{GHz}$. with $256 \mathrm{MB}$ Ram, connected through a 10/100 Mb. Router.

Figure 4 shows the service mean times for the 10 datasets, measuring the time required for the Buyer agents to buy their books within the book-trading e-market. For our conciliated approach, the graph shows the time spent for the contract-enforcing part only. The time spent in the first part of the process (obtaining a Working Team) was around 6 seconds. The mediated approach 
does not consider the time spent in looking for a mediator as it already knows it. These two aspects were not considered in the comparison as a roaming agent will usually carry out many transactions with the same MAS before leaving, hence these initial processes happens only once. In [4] a comparison of a typical closed contract-net-based MAS and our proposal is presented.

\section{Conclusions and Future Works}

An agent-based software architecture for allowing the dynamic participation of Foreign Agents into an existing MAS has been described. Additionally, an implementation has been carried out under the book-trading domain giving an insight on the viability of the proposal. The use of a mechanism based on dynamic behavior loading (conciliating differences at communication and semantic levels) raises as a feasible approach for obtaining an open MAS system.

Further work considers: extending to other levels of conciliation (e.g. communication protocol stack), different ways of services' delivery (e.g. codec, mediation, tuple-based) and applying our solution to other application domains such as transportation, robotics and supply-chain.

\section{Acknowledgement}

This work has been partially funded by CONICYT through Fondecyt Project No. 11080284 and the Pontificia Universidad Católica de Valparaíso (www.pucv.cl), through Nucleus Project No. 037.115/2008 "Collaborative Systems".

\section{Bibliography}

[1] G. Weiss. Multiagent Systems: A Modern Approach to Distributed Artificial Intelligence, MIT Press, Massachusetts, USA. 1999.

[2] M. Luck, P. McBurney, C. Preist, 2003. Agent Technology: Enabling Next Generation Computing. A Roadmap for Agent Based Computing, AgentLink II.

[3] P. Burrafato and M. Cossentino. Designing a multiagent solution for a bookstore with the passi methodology. In Fourth International Bi-Conference Workshop on Agent Oriented Information Systems (AOIS-2002).

[4] C. Cubillos, M. Donoso, 2009. Towards Open Agent Systems: A Book-Trading Study Case. In Fourth International Conference on Computer Sciences and Convergence Information Technology (ICCIT '09), pp. 950-953.

[5] C. Cubillos, M. Donoso, D. Cabrera-Paniagua, 2009. Designing an Open Agent System for Book-Trading. Third International Symposium on Intelligent Information Technology Application (IITA 2009), pp. 578-581.

[6] H. Lee, C. Chen, Nov. 2006. Multi-Agent Coalition Formation for Long-Term Task or Mobile Network. Int. Conf. on Computational Intelligence for Modelling, Control and Automation, 2006 and Int. Conf. on Intelligent Agents, Web Technologies and Internet Commerce, pp. $52-57$.

[7] M. Klusch, A. Gerber, 2002. Dynamic coalition formation among rational agents.IEEE Intelligent Systems, 17(3):42-47. 
[8] Foundation for Intelligent Physical Agents (FIPA). 2002. FIPA Interaction Protocols (IPs) Specifications. Available at: http://www.fipa.org/repository/ips.php3.

[9] Y.Labrou, T. Finin, Y. Peng, 1999. Agent communication languages: The current landscape.IEEE Intelligent Systems, 14(2), 45-52.

[10] Foundation of Intelligent Physical Agents (FIPA). 2002. FIPA ACL Message Structure Specification. Doc. No. SC00061g, 03/12/2002. Available at: "http://www.fipa.org/specs/fipa00061/"

[11] M. Uschold, R. Jasper and P. Clark. Three Approaches for Knowledge Sharing: A Comparative analysis. In Proceedings of the 12th Knowledge Acquisition, Modelling and Management Workshop, KAW'99, Banff, Canada, October 1999.

[12] FIPA Contract Net Interaction Protocol Specification. Available at: www.fipa.org/specs/fipa00029/SC00029H.pdf 